\title{
Point-Set Embeddings of Plane 3-Trees (Extended Abstract)
}

\author{
Rahnuma Islam Nishat, Debajyoti Mondal, and Md. Saidur Rahman \\ Graph Drawing and Information Visualization Laboratory, \\ Department of Computer Science and Engineering, Bangladesh University of \\ Engineering and Technology (BUET), Dhaka - 1000, Bangladesh \\ nishat.buet@gmail.com, debajyoti_mondal_cse@yahoo.com, \\ saidurrahman@cse.buet.ac.bd
}

\begin{abstract}
A straight-line drawing of a plane graph $G$ is a planar drawing of $G$, where each vertex is drawn as a point and each edge is drawn as a straight-line segment. Given a set $S$ of $n$ points on the Euclidean plane, a point-set embedding of a plane graph $G$ with $n$ vertices on $S$ is a straight-line drawing of $G$, where each vertex of $G$ is mapped to a distinct point of $S$. The problem of deciding if $G$ admits a point-set embedding on $S$ is NP-complete in general and even when $G$ is 2-connected and 2-outerplanar. In this paper we give an $O\left(n^{2} \log n\right)$ time algorithm to decide whether a plane 3 -tree admits a point-set embedding on a given set of points or not, and find an embedding if it exists. We prove an $\Omega(n \log n)$ lower bound on the time complexity for finding a pointset embedding of a plane 3-tree. Moreover, we consider a variant of the problem where we are given a plane 3 -tree $G$ with $n$ vertices and a set $S$ of $k>n$ points, and give a polynomial time algorithm to find a point-set embedding of $G$ on $S$ if it exists.
\end{abstract}

Keywords: Point-set embedding, Plane 3-tree, Lower bound.

\section{Introduction}

A straight-line drawing $\Gamma$ of a plane graph $G$ is a planar drawing of $G$, where each vertex is drawn as a point and each edge is drawn as a straight-line segment. The problem of computing a straight-line drawing of a graph where the vertices are constrained to be located at integer grid points is a classical problem in the graph drawing literature 612. One of the variants of this problem is to compute a planar embedding of a graph $G$ on a set of points $S$ where the points are located on the Euclidean plane 3911.

Let $G$ be a plane graph of $n$ vertices and $S$ be a set of $n$ points on the Euclidean plane. A point-set embedding of $G$ on $S$ is a straight-line drawing of $G$, where each vertex of $G$ is mapped to a distinct point of $S$. We do not restrict the points of $S$ to be in general position. In other words, three or more points in $S$ may be collinear. Figure 1(a) and (b) depict two sets $S$ and $S^{\prime}$ of 10 points, respectively. Figure 1(c) depicts a plane graph $G$ of 10 vertices. One can easily 
observe that $G$ admits a point-set embedding on $S^{\prime}$, as illustrated in Fig. 1(d). But $G$ does not admit a point-set embedding on $S$ since the convex hull of $S$ contains four points whereas the outer face of $G$ has three vertices.

A rich body of literature has been published on the point-set embeddings when the input graph $G$ is restricted to trees or outerplanar graphs [8]. Cabello [4] proved that the problem is NP-complete for planar graphs in general and even when the input graph is 2-connected and 2-outerplanar. Recently, Garcia et al. have given a characterization of a set of points $S$ such that there exists a 3connected cubic plane graph that admits a point-set embedding on $S$ [].

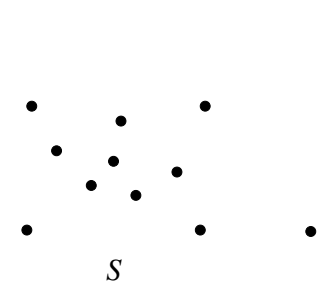

(a)

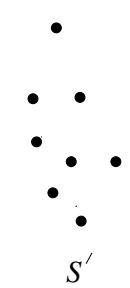

(b)

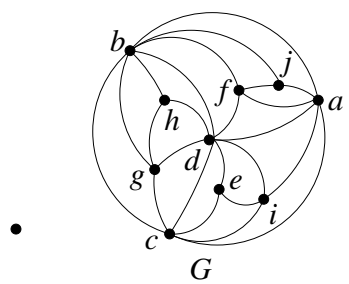

(c)

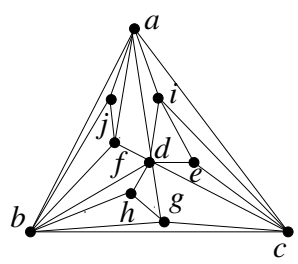

(d)

Fig. 1. (a) A set $S$ of 10 points, (b) a set $S^{\prime}$ of 10 points, (c) a plane graph $G$ of 10 vertices, and (d) a point-set embedding of $G$ on $S^{\prime}$

In this paper we consider the problem of obtaining point-set embeddings of "plane 3-trees". A plane 3-tree $G$ of $n \geq 3$ vertices is a plane graph for which the following (a) and (b) hold: (a) $G$ is a triangulated plane graph; (b) if $n>3$, then $G$ has a vertex whose deletion gives a plane 3 -tree $G^{\prime}$ of $n-1$ vertices. We give an $\mathrm{O}\left(n^{2} \log n\right)$ time algorithm that decides whether a plane 3-tree $G$ admits a point-set embedding on a given set $S$ of $n$ points or not; and computes a pointset embedding of $G$ if such an embedding exists. We prove an $\Omega(n \log n)$ lower bound on the time complexity for obtaining a point-set embedding of a plane 3 -tree of $n$ vertices on a set of $n$ points. Furthermore we give a polynomial-time algorithm to decide whether a plane 3 -tree $G$ of $n$ vertices admits a point-set embedding on a set of $k>n$ points.

The rest of this paper is organized as follows. Section 2 presents some definitions and preliminary results. Section 3 gives an $\mathrm{O}\left(n^{2} \log n\right)$ time algorithm to obtain a point-set embedding of a plane 3 -tree of $n$ vertices, if it exists. Section 4 shows an $\Omega(n \log n)$ lower bound on the running time for computing point-set embeddings of plane 3 -trees. Section 5 gives an $O\left(n k^{8}\right)$ time algorithm to decide whether a plane 3 -tree $G$ of $n$ vertices admits a point-set embedding on a set of $k>n$ points. Finally, Section 6 concludes the paper suggesting future works.

\section{Preliminaries}

In this section we give some relevant definitions that will be used throughout the paper and present some preliminary results. 
For the graph theoretic definitions which have not been described here, see [10].

A graph is planar if it can be embedded in the plane without edge crossings except at the vertices where the edges are incident. A plane graph is a planar graph with a fixed planar embedding. If all the faces of a plane graph $G$ are triangles, then $G$ is called a triangulated plane graph. A plane graph $G$ with $n \geq 3$ vertices is called a plane 3 -tree if the following (a) and (b) hold. (a) $G$ is a triangulated plane graph. (b) if $n>3$, then $G$ has a vertex whose deletion gives a plane 3 -tree $G^{\prime}$ of $n-1$ vertices. We denote by $G_{n}$ a plane 3 -tree of $n$ vertices. The following results are known on plane 3-trees [2].

Lemma 1. Let $G_{n}$ be a plane 3-tree of $n$ vertices where $n>3$. Then the following (a) and (b) hold. (a) $G_{n}$ has an inner vertex $x$ of degree three such that the removal of $x$ gives the plane 3 -tree $G_{n-1}$ of $n-1$ vertices. (b) $G_{n}$ has exactly one inner vertex $p$ which is the common neighbor of all the three outer vertices of $G_{n}$.

We call $p$ the representative vertex of $G_{n}$. Let $G$ be a plane graph. For a cycle $C$ in $G$, we denote by $G(C)$ the plane subgraph of $G$ inside $C$ (including $C$ ). A separating triangle of $G$ is a triangle in $G$ whose interior and exterior contain at least one vertex each. We now have the following lemma.

Lemma 2. Let $G_{n}$ be a plane 3-tree of $n>3$ vertices and $C$ be any triangle of $G_{n}$. Then the subgraph $G_{n}(C)$ is a plane 3 -tree.

Let $T$ be a rooted tree and $i$ be any vertex of $T$. Then we define a subtree $T(i)$ rooted at $i$ as a subgraph of $T$ induced by the vertex $i$ and all the descendants of $i$. An ordered rooted tree is a rooted tree where the children of any vertex are ordered counter-clockwise.

Let $G_{n}$ be a plane 3 -tree of $n$ vertices. Let $p$ be the representative vertex and $a, b, c$ be the outer vertices of $G_{n}$. The vertex $p$, along with the three outer vertices $a, b$ and $c$, form three triangles $a b p, b c p$ and cap. We call those three triangles the nested triangles around $p$. We now define a tree $T_{n-3}$ with the inner vertices of $G_{n}$ which we call the "representative tree" of $G_{n}$. The representative tree of $G_{n}$ is an ordered rooted tree $T_{n-3}$ satisfying the following two conditions (a) and $(b)$.

(a) $T_{n-3}$ consists of a single vertex if $n=3$.

(b) if $n>3$, then the root $p$ of $T_{n-3}$ is the representative vertex of $G_{n}$ and the subtrees rooted at the three counter-clockwise ordered children $p_{1}, p_{2}$ and $p_{3}$ of $p$ in $T_{n-3}$ are the representative trees of $G_{n}\left(C_{1}\right), G_{n}\left(C_{2}\right)$ and $G_{n}\left(C_{3}\right)$, respectively, where $C_{1}, C_{2}$ and $C_{3}$ are the three nested triangles around $p$ in counter-clockwise order.

Figure2(a) depicts a plane 3 -tree $G_{n}$ and Fig. 2(b) depicts the representative tree $T_{n-3}$ of $G_{n}$. We now have the following lemma whose proof has been omitted.

Lemma 3. Let $G_{n}$ be any plane 3 -tree of $n \geq 3$ vertices. Then $G_{n}$ has a unique representative tree $T_{n-3}$ with exactly $n-3$ internal vertices and $2 n-5$ leaves. Moreover, $T_{n-3}$ can be found in time $O(n)$. 


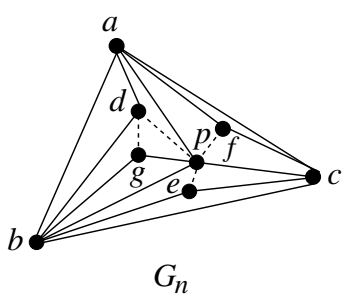

(a)

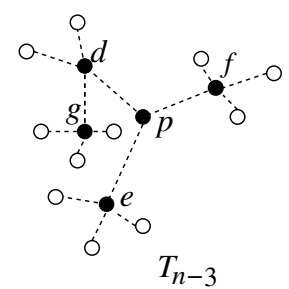

(b)

Fig. 2. (a) A plane 3-tree $G_{n}$ and (b) the representative tree $T_{n-3}$ of $G_{n}$

We now have the following lemma whose proof is immediate from the definition of the representative tree and Lemma 3.

Lemma 4. Let $T_{n-3}$ be the representative tree of a plane 3 -tree $G_{n}$ of $n \geq 3$ vertices and let $T(i)$ be an ordered subtree rooted at a vertex $i$ of $T_{n-3}$. Then there exists a unique triangle $C$ in $G_{n}$ such that $T(i)$ is the representative tree of $G_{n}(C)$.

By Lemma 4 , for any vertex $p$ of $T_{n-3}$, there is a unique triangle in $G_{n}$ which we denote as $C_{p}$. For the rest of this paper, we shall often use an internal vertex $p$ of $T_{n-3}$ and the representative vertex of $G_{n}\left(C_{p}\right)$ interchangeably.

\section{Point-Set Embeddings of Plane 3-Trees}

In this section we give an $\mathrm{O}\left(n^{2} \log n\right)$ time algorithm to decide whether a plane 3 -tree $G$ of $n$ vertices has a point-set embedding on a set $S$ of $n$ points or not, and obtain a point-set embedding of $G$ on $S$ if it exists.

Before presenting the detail of our algorithm we focus on some properties of the point-set embeddings of plane 3-trees. Let $S$ be a set of $n$ points on the Euclidean plane. The convex-hull of $S$ is the smallest convex polygon that encloses all the points in $S$. Let $G$ be a plane 3 -tree of $n$ vertices. In any pointset embedding of $G$ the outer face of $G$ is drawn as a triangle and hence the following fact holds.

Fact 1. Let $G$ be a plane 3 -tree of $n$ vertices and $S$ be a set of $n$ points. If $G$ admits a point-set embedding on $S$, then the convex-hull of $S$ contains exactly three points in $S$.

By Fact 1, $G$ has no point-set embedding on $S$ if the convex-hull of $S$ does not contain exactly three points in $S$. We thus assume that the convex-hull of $S$ contains exactly three points. One may observe that the outer vertices of $G$ can be mapped to the three points on the convex-hull of $S$ in six ways, and hence we need to check whether $G$ admits a point-set embedding on $S$ or not for each of the six mappings. In the remaining of this section we give an algorithm to check whether $G$ admits a point-set embedding on $S$ for a given mapping of the outer vertices of $G$ to the three points of the convex-hull of $S$. 
Let $T_{n-3}$ be the representative tree of $G$ and let $p$ be the root of $T_{n-3}$. Let $C_{p_{1}}, C_{p_{2}}, C_{p_{3}}$ be the three nested triangles around $p$. By lemma $2, G_{n}\left(C_{p_{1}}\right)$, $G_{n}\left(C_{p_{2}}\right)$ and $G_{n}\left(C_{p_{3}}\right)$ are three plane 3-trees which have the corresponding unique representative trees $T_{n_{1}}\left(p_{1}\right), T_{n_{2}}\left(p_{2}\right)$ and $T_{n_{3}}\left(p_{3}\right)$ where $n_{1}, n_{2}$ and $n_{3}$ are the number of internal vertices of $T_{n_{1}}\left(p_{1}\right), T_{n_{2}}\left(p_{2}\right)$ and $T_{n_{3}}\left(p_{3}\right)$, respectively. Let $C$ be a cycle in $G$. We denote by $\Gamma(C)$ the embedding of $C$ on some points of $S$. We call a mapping of $p$ to a point $x \in S$ a valid mapping of $p$ if the proper interiors of $\Gamma\left(C_{p_{1}}\right), \Gamma\left(C_{p_{2}}\right)$ and $\Gamma\left(C_{p_{3}}\right)$ contain $n_{1}, n_{2}$ and $n_{3}$ points, respectively. We now have the following lemma whose proof is omitted in this extended abstract.

Lemma 5. Let $G$ be a plane 3-tree of $n$ vertices, let $a, b$ and $c$ be the three outer vertices of $G$, and let $p$ be the representative vertex of $G$. Let $S$ be a point set of $n$ points such that the convex hull of $S$ contains exactly three points. Assume that $G$ has a point-set embedding $\Gamma(G)$ on $S$ for a given mapping of $a, b$ and $c$ to the three points of the convex-hull of $S$. Then $p$ has a unique valid mapping for the given mapping of $a, b$ and $c$.

Based on Fact 1 and Lemma 5 we now describe an algorithm to find a point-set embedding of a plane 3-tree $G$ on a point-set $S$. The algorithm first computes the convex-hull of $S$ and then recursively finds valid mappings of the representative vertices. A naive approach to find a valid mapping of a representative vertex $p$ is as follows. For each point $u \in S$ interior to $\Gamma\left(C_{p}\right)$, we assume $u$ as the representative vertex and check whether $\Gamma\left(C_{p_{1}}\right), \Gamma\left(C_{p_{2}}\right)$ and $\Gamma\left(C_{p_{3}}\right)$ contains the required number of points. One can easily compute a valid mapping of $p$ in $O\left(n^{2}\right)$ time. Now, if we compute the mappings of the representative vertices for the plane 3 -trees $G_{n}\left(C_{p_{1}}\right), G_{n}\left(C_{p_{2}}\right)$ and $G_{n}\left(C_{p_{3}}\right)$ in a recursive fashion, we can obtain a point-set embedding of $G$. Since there are $n-3$ representative vertices, computation of the final embedding takes $O(n) \times O\left(n^{2}\right)=O\left(n^{3}\right)$ time. We call this algorithm Point-set-embedding, and in the rest of this section we give a faster method to find a valid mapping of a representative vertex and use it to implement Algorithm Point-set-embedding in $O\left(n^{2} \log n\right)$ time.

We first compute a convex-hull of $S$ in $O(n \log h)$ time [5], where $h$ is the number of points on the hull. If $G$ admits a point-set embedding on $S$, then the convex-hull contains exactly three points in $S$. For a given mapping of the three outer vertices $a, b, c$ of $G$ to the three points on the convex hull of $S$, we sort the points interior to the triangle $a b c$ by increasing polar angle around $a$ in clockwise order. Then we put the sorted list in an array $A_{a}$ as illustrated in Fig. 3 . For the points with the same slope, we keep a pointer to the point closest to $a$ from the other points with the same slope. The point closest to $a$ is the parent of the other points with the same slope. Let $x$ and $y$ be any two points where $y$ has a pointer to $x$. Then $y$ is a child of $x$. We also maintain a count of the children of a parent in $A_{a}$. In a similar way we construct $A_{b}$ and $A_{c}$ for $b$ and $c$, respectively. Clearly, the construction of these three arrays takes $O(n \log n)$ time.

We next take any point $u \in S$, interior to the triangle $a b c$, and draw straight lines through $(u, a),(u, b)$ and $(u, c)$ which intersect $b c, a c, a b$ at $p, q$ and $r$, respectively as illustrated in Fig 4. Thus the region inside the triangle $a b c$ gets 


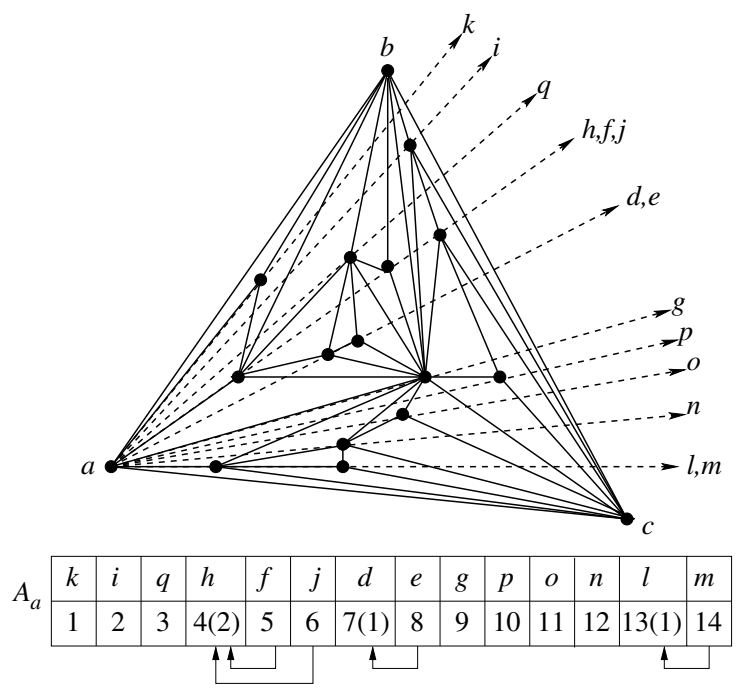

Fig. 3. Illustration for the construction of the array $A_{a}$

split into six disjoint regions which we denote as $x_{1}, x_{2}, \ldots, x_{6}$. The regions $x_{1}$, $x_{2}, x_{3}, x_{4}, x_{5}, x_{6}$ are bounded by the triangles aur, auq, cuq, cup, bup, bur, respectively. Moreover, by $x_{7}, x_{8}$ and $x_{9}$ we denote the three lines shared by region $x_{1}$ and $x_{6}, x_{2}$ and $x_{3}, x_{4}$ and $x_{5}$, respectively. In the remaining of this section we also denote by $x_{i}, 1 \leq i \leq 9$, the number of points of $S$ in the region $x_{i}$.

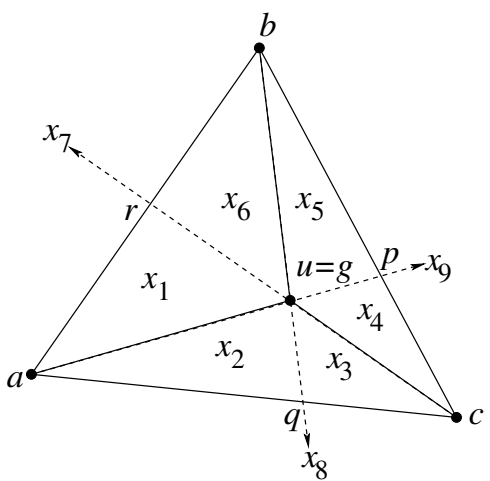

Fig. 4. Computation of the mapping of the representative vertex

Let $T_{n-3}$ be the representative tree of $G$ and $p$ be the root of $T_{n-3}$. Let $n_{1}, n_{2}$ and $n_{3}$ be the number of vertices of the three subtrees rooted at the three children of $p$. We now formulate a set of nine linear equations and solve them to check whether $u$ is a valid mapping for $p$. The three constraints are $x_{2}+x_{8}+x_{3}=n_{1}$, $x_{4}+x_{9}+x_{5}=n_{2}$ and $x_{1}+x_{7}+x_{6}=n_{3}$ which can be obtained easily. For example, 
the constraints are $x_{2}+x_{8}+x_{3}=4, x_{4}+x_{9}+x_{5}=3$ and $x_{1}+x_{7}+x_{6}=6$ for the graph of Fig. 3 .

The nine equations can be obtained using the three straight lines $(u, a),(u, b)$ and $(u, c)$ as follows. The straight line $(u, a)$ splits the triangle $a b c$ into two disjoint regions $x_{1}+x_{5}+x_{6}+x_{7}$ and $x_{2}+x_{3}+x_{4}+x_{8}$. The number of points in those regions are $x_{1}+x_{5}+x_{6}+x_{7}=u_{i}-1$ and $x_{2}+x_{3}+x_{4}+x_{8}=\left|A_{a}\right|-u_{i}-u_{a}$ where $u_{i}$ is the index of $u$ in $A_{a}$ and $u_{a}$ is the count of children of $u$ in $A_{a}$. The number of points on $x_{9}$ is equal to $u_{a}$ which gives another equation $x_{9}=u_{a}$. Let $u_{j}$ and $u_{k}$ be the indices of a point $u$ in $A_{b}$ and $A_{c}$, respectively and let $u_{b}$ and $u_{c}$ be the counts of children of $u$ in $A_{b}$ and $A_{c}$, respectively. Then we can derive six other equations using $(u, b)$ and $(u, c)$ in a similar way as described above. The nine equations for any point $u$, interior to the triangle $a b c$, are $x_{1}+x_{5}+x_{6}+x_{7}=$ $u_{i}-1, x_{2}+x_{3}+x_{4}+x_{8}=\left|A_{a}\right|-u_{i}-u_{a}, x_{9}=u_{a}, x_{5}+x_{9}+x_{4}+x_{3}=u_{j}-1$, $x_{2}+x_{1}+x_{7}+x_{6}=\left|A_{b}\right|-u_{j}-u_{b}, x_{8}=u_{b}, x_{3}+x_{8}+x_{2}+x_{1}=u_{k}-1$, $x_{4}+x_{9}+x_{5}+x_{6}=\left|A_{c}\right|-u_{k}-u_{c}$ and $x_{7}=u_{c}$. When the vertex $g$ is mapped to the point $u$ in the graph of Fig. 3 , the equations become $x_{1}+x_{5}+x_{6}+x_{7}=8$, $x_{2}+x_{3}+x_{4}+x_{8}=5, x_{9}=0, x_{5}+x_{9}+x_{4}+x_{3}=3, x_{2}+x_{1}+x_{7}+x_{6}=10$, $x_{8}=0, x_{3}+x_{8}+x_{2}+x_{1}=7, x_{4}+x_{9}+x_{5}+x_{6}=5$ and $x_{7}=1$.

If we get a unique solution of the set of linear equations, then $u$ is a valid mapping of $p$ by Lemma 5. A simple "Gaussian elimination" to solve a system of $t$ equations for $t$ unknowns requires $O\left(t^{3}\right)$ time. Here $t=9$ and hence we can verify whether $u$ is a valid mapping of $p$ in $O(1)$ time. Similarly we check the points inside the triangle $a b c$, other than $u$, to obtain a valid mapping of $p$. Since there are $O(n)$ points inside the triangle $a b c$, this step takes $O(n) \times O(1)=O(n)$ time.

Finally, we find the valid mappings of representative vertices for the smaller plane 3 -trees in a recursive fashion and obtain a point-set embedding of $G$. At each recursive step we construct three sorted arrays in $O(n \log n)$ time and find the mapping of the representative vertex in $O(n)$ time. Since there are $O(n)$ representative vertices, computation of the final embedding takes $O(n) \times$ $(O(n \log n)+O(n))=O\left(n^{2} \log n\right)$ time. We now recall that the computation of the convex-hull takes $O(n \log h)$ time and there are six ways of mapping the three outer vertices of $G$ to the three points on the convex-hull of $S$. Therefore the time taken for deciding whether $G$ admits a point-set embedding on $S$ is $\left(O(n \log h)+6 \times O\left(n^{2} \log n\right)\right)=O\left(n^{2} \log n\right)$ time. We now have the following theorem.

Theorem 1. Given a plane 3-tree $G$ of $n$ vertices and a point-set $S$ of $n$-points, Algorithm Point-set-embedding computes a point-set embedding of $G$ on $S$ in $O\left(n^{2} \log n\right)$ time if such an embedding exists.

One can observe that the bottle-neck of Algorithm Point-set-embedding is to construct three sorted arrays at each recursive step; which takes $O(n \log n)$ time at each step and $O\left(n^{2} \log n\right)$ time in total. But if we assume that the points are in general position, we can construct the sorted arrays for all the points collectively at the initial step of the algorithm in $O\left(n^{2}\right)$ time using "arrangements" [1]. At each recursive step we can update those arrays in such a way that the total 
number of updates after all the recursive steps becomes $O\left(n^{2}\right)$. Thus, when the points are assumed to be in general position, the time required to decide whether $G$ admits a point-set embedding on $S$ becomes $O\left(n^{2}\right)+O(n \log h)+6 \times O\left(n^{2}\right)=$ $O\left(n^{2}\right)$.

\section{Lower Bound}

In this section, we first analyze the time complexity of Algorithm Point-SetEmbedding in some restricted cases. We then show that the lower bound on time complexity for computing a point-set embedding of a plane 3-tree of $n$ vertices is $\Omega(n \log n)$.

Let $G$ be a plane 3-tree of $n$-vertices and let $T$ be the representative tree of $G$. Suppose that, for each internal vertex $u$ of $T$ the ratio of the number of vertices in the three subtrees rooted at three children of $u$ is $x: y: z$. Without loss of generality we assume that $x \geq y \geq z$. One can easily find that running time of the Algorithm Point-Set-Embedding can be written as $O(n)+T(n)$ where the term $O(n)$ is to obtain the embedding of the outer face of $G$ and the term $T(n) \geq T(n-3)$ is the time to obtain the embedding of $n-3$ internal vertices of $G$. Since at each recursive step we take $O(n \log n)$ time to obtain a valid mapping of a representative vertex, $T(n)$ can be defined recursively as follows. $T(n) \leq T\left(\frac{n x}{x+y+z}\right)+T\left(\frac{n y}{x+y+z}\right)+T\left(\frac{n z}{x+y+z}\right)+c n \log n=3 T\left(\frac{n}{b}\right)+c n \log n$. Here $c$ is a constant hidden in $O(n \log n)$ term and $b=\frac{x+y+z}{x}$. We observe that, for $b=\sqrt{ } 3, T(n)=3 T\left(\frac{n}{\sqrt{ } 3}\right)+c n \log n=O\left(n^{2}\right) ;$ for $b=2, T(n)=3 T\left(\frac{n}{2}\right)+c n \log n=$ $O\left(n^{1.58}\right)$; and for $b=3, T(n)=3 T\left(\frac{n}{3}\right)+c n \log n=O\left(n \log ^{2} n\right)$. Therefore, we obtain the following theorem.

Theorem 2. Let $G$ be a plane 3-tree of $n$ vertices and $S$ be a point-set of $n$ points. If the representative tree of $G$ is a complete ternary tree, it can be decided whether $G$ admits a point-set embedding on $S$ in $O\left(n \log ^{2} n\right)$ time.

We now prove a lower bound on the running time of the problem of obtaining a point-set embedding of a plane 3-tree with $n$ vertices as in the following theorem.

Theorem 3. The lower bound on the running time of the problem of computing point-set embeddings of plane 3 -trees with $n$ vertices is $\Omega(n \log n)$.

Proof. We reduce sorting problems into the problem of computing point-set embeddings of plane 3 -trees in the sense that point-set embedding algorithm can be used to solve sorting problems with little additional work.

Let $L=\left(x_{1}, x_{2}, \ldots, x_{n}\right)$ be a list of $n$ unsorted numbers to be sorted and let the smallest number in $L$ be $x_{\text {min }}$. Without loss of generality we assume that $x_{i}>0,1 \leq i \leq n$, since if there exists an $x_{i} \leq 0$ we can obtain a list $L^{\prime}$ of nonzero positive numbers by adding $1-x_{\min }$ to all the $x_{i}, 1 \leq i \leq n$. One can observe that the sorted order of the numbers in $L^{\prime}$ yields a sorted order of the numbers in $L$. Suppose that we have an algorithm $\mathbf{X}$ that computes a point-set embedding of a plane 3 -tree of $n$ vertices in $f(n)$ time. We show that Algorithm 
$\mathbf{X}$ can be used to solve a sorting problem of $n$ numbers in time $f(n)+O(n)$ where the $O(n)$ represents additional time to convert the solution of $\mathbf{X}$ to the solution of the sorting problem.

Let $x_{\max }$ be the maximum number in $L$ which can be found in $O(n)$ time. We make a set $S$ of two-dimensional points $\left(x_{i}, x_{i}^{2}\right) ; 1 \leq i \leq n$ and let $S^{\prime}=$ $S \cup\left\{\left(x_{\max }+1,0\right),(0,0)\right\}$. Let $G$ be a plane 3 -tree of $n+2$ vertices such that its representative tree $T$ has the following properties (a) and (b). (a) The left child and the right child of each internal vertex of $T$ are leaves. (b) The subgraph induced by the internal vertices of $T$ is a path of $n-1$ vertices. Figure 5(a), (b) and (c) illustrate $S^{\prime}, G$ and $T$, respectively. We now use Algorithm $\mathbf{X}$ to compute a point-set embedding of $G$ on $S$.

In a point-set embedding $\Gamma(G)$ of $G$ on $S^{\prime}$, the outer vertices of $G$ is mapped to the convex-hull of $S^{\prime}$ by Fact 1 . The convex-hull of $S^{\prime}$ contains the points $(0,0)$, $\left(x_{\max }+1,0\right)$ and $\left(x_{\max }, x_{\max }^{2}\right)$ which we denote by $a, b$ and $c$, respectively. Let the representative vertex $p$ of $G$ be mapped to a point $z \in S^{\prime}$ in $\Gamma(G)$ and let the proper interiors of the triangles $a b z=C_{p_{1}}, b c z=C_{p_{2}}, c a z=C_{p_{3}}$ be $R_{z_{1}}, R_{z_{2}}$, $R_{z_{3}}$, respectively. Since $p$ is also the root of $T$ with two leaves, two of the regions $R_{z_{1}}, R_{z_{2}}$ and $R_{z_{3}}$ do not contain any point of $S$. One can observe that such two regions can be obtained if $z$ is the second smallest (or the second largest) number of $L$. Suppose that the region $R_{z_{2}}$ (or $R_{z_{1}}$ ) contains all the points of $S^{\prime}$ other than $a, b, c$ and $z$. We now consider the point-set embedding of $G\left(C_{p_{2}}\right)$ (or $G\left(C_{p_{1}}\right)$ ). Let $c_{1}, c_{2}$ and $c_{3}$ be the left, middle and right child of $p$ in $T$. If the children are leaves, we have no vertices left to consider. Otherwise $c_{2}$ is an internal vertex. Since the left child and the right child of $c_{1}$ are leaves, $c_{1}$ must be mapped to the next smallest number or (next largest number) to ensure two regions which do not contain any points of $S^{\prime}$, maintaining the plane embedding of $G$.

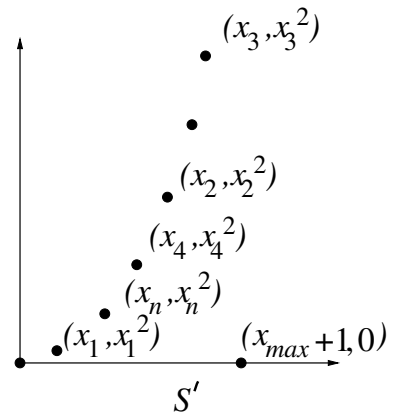

(a)

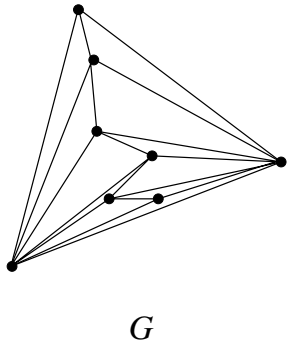

(b)

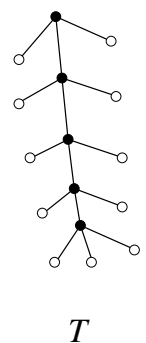

(c)

Fig. 5. Illustration for the proof of Theorem 3

Thus the sequence of $x$-coordinates of the mappings of the internal vertices of $T$ from the root gives an increasing (or decreasing) order of the numbers in $L-\left\{x_{\max }\right\}$. Moreover, we can check whether the obtained order is increasing or decreasing and place $x_{\max }$ in the correct position in constant time. Thus, we 
can use Algorithm $\mathbf{X}$ to solve the sorting problem which implies that the lower bound of Algorithm $\mathbf{X}$ is equal to the lower bound $\Omega(n \log n)$ on the running time of sorting problems.

\section{Generalized Case}

In this section we consider the problem of computing a point-set embedding of a plane 3-tree $G$, when the number of given points is greater than the number of vertices of $G$. We use dynamic programming to solve the problem.

Let $G$ be a plane 3 -tree of $n$ vertices and $S$ be a point-set of $k>n$ points. We obtain a grid by drawing a vertical line and a horizontal line passing through each of the $k$ points. We assign $x$-coordinates for the vertical lines according to the count from left to right. Similarly we assign $y$-coordinates for the horizontal lines according to the count from bottom to top. The grid points are the intersections of the horizontal and the vertical lines. A valid grid point is a grid point which is also a point in $S$. The width $W$ and height $H$ of the grid are measured by the width and the height of the smallest rectangle with sides parallel to the axes which encloses all the points in $S$. The size of the grid is usually described as $W \times H$. Since the number of points in $S$ is $k$, the size of the grid is at most $k \times k$. A graph $G$ is drawable on a grid obtained from a point-set $S$ if $G$ admits a straight-line drawing such that the vertices of $G$ are mapped on to the valid points on the grid obtained from $S$.

We assume a height $h$ and a width $w$, iterate $h$ from 1 to $k$ and for each $h$, we iterate $w$ from 1 to $k$. At each iteration we check whether $G$ is drawable on a $w \times h$ grid or not. We give two algorithms Algorithm Point-Set and Algorithm Feasibility-Checking to serve this purpose. Let $T$ be the representative tree of $G$. At each iteration we traverse $T$ in preorder. For each internal vertex $i$ of $T$, Algorithm Point-Set generates all possible $(x, y)$-coordinate assignments for the outer vertices $a, b$ and $c$ of $G\left(C_{i}\right)$ within area $w \times h$. For each such $(x, y)$-coordinate assignment of $a, b$ and $c$, Algorithm Feasibility-Checking is called to check whether $G\left(C_{i}\right)$ is drawable. Here we formally define the input and output of the problem Feasibility-Checking.

Input: A plane 3-tree $G$ and a mapping of the three outer vertices $a, b$ and $c$ of $G$ to the three different valid points on a grid obtained from $S$.

Output: If $G$ is drawable with the given mapping of $a, b$ and $c$, then the output is True. Otherwise, the output is False.

We denote the $x$-coordinate and $y$-coordinate of a vertex $v$ by $v_{x}$ and $v_{y}$, respectively. We denote by $F_{p}\left(a_{y}^{x}, b_{y}^{x}, c_{y}^{x}\right)$ the Feasibility Checking problem of any vertex $p$ of $T$ where $a_{y}^{x}, b_{y}^{x}, c_{y}^{x}$ are the $(x, y)$-coordinates of the three outer vertices $a, b$ and $c$ of $G\left(C_{p}\right)$. We solve this decision problem by showing in Lemma 6 that the optimal solution of the problem consists of the optimal solutions of the subproblems. Theorem 4 states the recursive solution of the Feasibility Checking problem. 
Lemma 6. Let $G$ be a plane 3-tree with the representative tree $T$. Let $p$ be any internal vertex of $T$ with the three children $p_{1}, p_{2}, p_{3}$ in $T$ and $a, b, c$ be the outer vertices of $G\left(C_{p}\right)$. Then, for an assignment of the vertices $a, b, c$ and $p$ to valid grid points, the Feasibility Checking problems of $p_{1}, p_{2}$ and $p_{3}$ are the three subproblems of the Feasibility Checking problem of $p$.

Theorem 4. Let $G$ be a plane 3-tree with the representative tree $T$ and $p$ be any vertex of $T$. Let $a, b, c$ be the three outer vertices of $G\left(C_{p}\right)$ and $p_{1}, p_{2}, p_{3}$ be the three children of $p$ when $p$ is an internal vertex of $T$. Let $F_{p}\left(a_{y}^{x}, b_{y}^{x}, c_{y}^{x}\right)$ be the Feasibility Checking problem of $p$. Then $F_{p}\left(a_{y}^{x}, b_{y}^{x}, c_{y}^{x}\right)$ has the following recursive formula.

$$
F_{p}\left(a_{y}^{x}, b_{y}^{x}, c_{y}^{x}\right)=\left\{\begin{array}{c}
\text { False if }\left(\max \left\{a_{x}, b_{x}, c_{x}\right\}-\min \left\{a_{x}, b_{x}, c_{x}\right\}=0\right) \\
\vee\left(\max \left\{a_{y}, b_{y}, c_{y}\right\}-\min \left\{a_{y}, b_{y}, c_{y}\right\}=0\right) ; \\
\text { True if }\left(\max \left\{a_{x}, b_{x}, c_{x}\right\}-\min \left\{a_{x}, b_{x}, c_{x}\right\} \geq 1\right) \\
\wedge\left(\max \left\{a_{y}, b_{y}, c_{y}\right\}-\min \left\{a_{y}, b_{y}, c_{y}\right\} \geq 1\right) \\
\wedge p \text { is a leaf; } \\
\text { False if } p \text { is an internal vertex and there is no valid } \\
\text { grid point inside the triangle abc; } \\
\left.F_{p_{x}, p_{y}}\left(a_{y}^{x}, b_{y}^{x}, p_{y}^{x}\right) \wedge F_{p_{2}}\left(b_{y}^{x}, c_{y}^{x}, p_{y}^{x}\right) \wedge F_{p_{3}}\left(c_{y}^{x}, a_{y}^{x}, p_{y}^{x}\right)\right\} \\
\text { where }\left(p_{x}, p_{y}\right) \text { is a valid grid point inside } \\
\text { the triangle abc, otherwise. }
\end{array}\right.
$$

Proof. First we consider the case when $\left(\max \left\{a_{x}, b_{x}, c_{x}\right\}-\min \left\{a_{x}, b_{x}, c_{x}\right\}=0\right) \vee$ $\left(\max \left\{a_{y}, b_{y}, c_{y}\right\}-\min \left\{a_{y}, b_{y}, c_{y}\right\}=0\right)$. Then we assign $F_{p}\left(a_{y}^{x}, b_{y}^{x}, c_{y}^{x}\right)=$ False because a grid of at least area $1 \times 1$ is necessary to draw a triangle. The next case is $\left(\max \left\{a_{x}, b_{x}, c_{x}\right\}-\min \left\{a_{x}, b_{x}, c_{x}\right\} \geq 1\right) \wedge\left(\max \left\{a_{y}, b_{y}, c_{y}\right\}-\min \left\{a_{y}, b_{y}, c_{y}\right\} \geq 1\right)$ when $p$ is a leaf. Then we assign $F_{p}\left(a_{y}^{x}, b_{y}^{x}, c_{y}^{x}\right)=$ True since area $1 \times 1$ is sufficient to draw a triangle. In the next case, $p$ is an internal vertex and there is no valid grid point inside the triangle $a b c$. We assign $F_{p}\left(a_{y}^{x}, b_{y}^{x}, c_{y}^{x}\right)=$ False since $p$ cannot be placed inside $C_{p}$. The remaining case is $\max \left\{a_{x}, b_{x}, c_{x}\right\}-\min \left\{a_{x}, b_{x}, c_{x}\right\}>$ 1) $\wedge\left(\max \left\{a_{y}, b_{y}, c_{y}\right\}-\min \left\{a_{y}, b_{y}, c_{y}\right\}>1\right)$ when $p$ is an internal vertex. Then we define $F_{p}\left(a_{y}^{x}, b_{y}^{x}, c_{y}^{x}\right)$ recursively according to Lemma 6 .

One can associate tables to store the computed $(x, y)$-coordinates of the vertices of $G$ to obtain the point-set embedding of $G$ in polynomial time, if it exists.

Theorem 5. Given a plane 3 -tree $G$ with $n \geq 3$ vertices and a point-set of $k>n$ points, Algorithm Point-Set decides whether $G$ admits a point-set embedding on $S$ in time $O\left(n k^{8}\right)$.

\section{Conclusion}

We give an $\mathrm{O}\left(n^{2} \log n\right)$ time algorithm that decides whether a plane 3 -tree $G$ of $n$-vertices admits a point-set embedding on a set $S$ of $n$ points or not; and computes a point-set embedding of $G$ if such an embedding exists. We observe that it is possible to obtain a point-set embedding of $G$ on $S$ in $O\left(n^{2}\right)$ time 
if the points are assumed to be in general position. We prove an $\Omega(n \log n)$ lower bound on the time complexity for obtaining point-set embeddings of plane 3 -trees. Beside obtaining a point-set embedding of a plane 3-tree, we consider a generalized problem when the given point-set has more than $n$ points and give a polynomial-time algorithm to solve the problem. It is a challenge to find simpler algorithms for obtaining point-set embeddings of plane 3-trees both in the restricted and generalized cases.

\section{Acknowledgment}

This work is done in Graph Drawing \& Information Visualization Laboratory of the Department of CSE, BUET established under the project "Facility Upgradation for Sustainable Research on Graph Drawing \& Information Visualization" supported by the Ministry of Science and Information \& Communication Technology, Government of Bangladesh. We thank Tanaeem Muhammad Moosa for fruitful discussions.

\section{References}

1. de Berg, M., van Kreveld, M., Overmars, M., Schwarzkopf, O.: Computational Geometry: Algorithms and Applications. Springer, Heidelberg (2000)

2. Biedl, T., Velázquez, L.E.R.: Drawing planar 3-trees with given face-areas. In: Eppstein, D., Gansner, E.R. (eds.) GD 2009. LNCS, vol. 5849, pp. 316-322. Springer, Heidelberg (2010)

3. Bose, P.: On embedding an outer-planar graph in a point set. Computational Geometry - Theory and Applications 23(3), 303-312 (2002)

4. Cabello, S.: Planar embeddability of the vertices of a graph using a fixed point set is NP-hard. Journal of Graph Algorithms and Applications 10(2), 353-363 (2006)

5. Chan, T.M.: Optimal output-sensitive convex hull algorithms in two and three dimensions. Discrete \& Computational Geometry 16(4), 361-368 (1996)

6. de Fraysseix, H., Pach, J., Pollack, R.: How to draw a planar graph on a grid. Combinatorica 10, 41-51 (1990)

7. García, A., Hurtado, F., Huemer, C., Tejel, J., Valtr, P.: On embedding triconnected cubic graphs on point sets. Electronic Notes in Discrete Mathematics 29, 531-538 (2007)

8. Ikebe, Y., Perles, M.A., Tamura, A., Tokunaga, S.: The rooted tree embedding problem into points in the plane. Discrete \& Computational Geometry 11, 51-63 (1994)

9. Kaufmann, M., Wiese, R.: Embedding vertices at points: Few bends suffice for planar graphs. Journal of Graph Algorithms and Applications 6(1), 115-129 (2002)

10. Nishizeki, T., Rahman, M.S.: Planar Graph Drawing. World Scientific, Singapore (2004)

11. Pach, J., Gritzmann, P., Mohar, B., Pollack, R.: Embedding a planar triangulation with vertices at specified points. American Mathematical Monthly 98, 165-166 (1991)

12. Schnyder, W.: Embedding planar graphs on the grid. In: The first annual ACMSIAM symposium on Discrete algorithms, pp. 138-148 (1990) 